\title{
Martine Sonnet, Brigitte Keriven, Claude Ghiati, Isabelle Havelange, Bibliographie annuelle de l'histoire de France du Ve siècle à 1958. Année 2000
}

\section{Bernard Merdrignac}

\section{(2) OpenEdition \\ 1 Journals}

\section{Édition électronique}

URL : http://journals.openedition.org/abpo/1588

DOI : $10.4000 /$ abpo. 1588

ISBN : 978-2-7535-1487-4

ISSN : 2108-6443

\section{Éditeur}

Presses universitaires de Rennes

\section{Édition imprimée}

Date de publication : 20 septembre 2002

Pagination : $174-176$

ISBN : 978-2-86847-768-2

ISSN : 0399-0826

\section{Référence électronique}

Bernard Merdrignac, "Martine Sonnet, Brigitte Keriven, Claude Ghiati, Isabelle Havelange, Bibliographie annuelle de l'histoire de France du Ve siècle à 1958. Année 2000 ", Annales de Bretagne et des Pays de l'Ouest [En ligne], 109-3 | 2002, mis en ligne le 20 septembre 2004, consulté le 23 septembre 2020. URL : http://journals.openedition.org/abpo/1588; DOI : https://doi.org/10.4000/abpo.1588 
172) etc. À la même rubrique de la "spiritualité catholique ", sont évoquées les nombreuses " apparitions mariales " (p. 16), qui donnent elles-mêmes naissance aux " congrégations mariales" (p. 70), placées sous l'autorité des Jésuites et héritières de la reconquête catholique du $\mathrm{xVI}^{\mathrm{e}}$ siècle. Ainsi, la lecture attentive de ce dictionnaire permet de renouer le lien chronologique et intellectuel entre la France d'ancien régime et celle de l'âge industriel dans la mesure où l'on peut mieux mesurer le poids des continuités, évident dans le cas des formes de la pratique religieuse et l'importance de la dévotion mariale, illustrée par l'information mentionnée ici que le Traité de la vraie dévotion à la Sainte Vierge de Grignion de Montfort, composé en 1712, a été publié en 1842.

Les questions politiques, particulièrement importantes dans ce " siècle des révolutions ", sont largement traitées, grâce aux entrées chronologiques par type de régime évoquées plus haut, mais par la rubrique thématique portant sur les " constitutions ": de 1791 à 1875, pas moins de huit textes fondamentaux déterminent l'organisation des pouvoirs dans le pays, sur lesquels il fallait faire le point de façon précise et synthétique. On regrettera cependant que, dans le corps de la notice (p. 73-75), l'occasion ne se soit pas présentée de faire figurer le mot " charte" , ce qui aurait permis de renvoyer aux notices " charte", de 1814 et de 1830 (p. 52-53) afin de permettre à l'étudiant soucieux de repérage chronologique de bien s'y retrouver dans la succession des régimes politiques que la France a connus. Lorsque la faillite des régimes constitutionnels est patente, il arrive qu'un coup d'État les renverse : si les limites chronologiques imposées à cet ouvrage n'ont pas permis aux auteurs de prévoir une notice sur le coup d'État de brumaire (mentionné, mais sans renvoi, dans le corps de la notice sur le Consulat, p. 75), à l'inverse, le coup d'État du 2 décembre 1851 et ses conséquences immédiates donne lieu à une notice extrêmement précise (p. 78-79). La liste exhaustive qui a été établie des gouvernements de la France, de 1799 à 1914, avec l'indication des noms de quelques uns des titulaires des portefeuilles ministériels, sera d'une très grande utilité pour tous les étudiants confrontés aux explications de textes historiques (p. 125-130).

Quant à la "révolution industrielle ", notion de plus en plus difficile à définir au fur et à mesure de l'avancement des recherches, elle est traitée dans une notice (p. 224) qui renvoie elle-même à une autre brève rubrique, la " protoindustrialisation " (p. 208). Ces deux articles, qui ont le mérite de montrer de façon simple pourquoi ce concept traditionnel est largement remis en cause, renvoient à des secteurs de développement économique (la sidérurgie) mais pas aux structures du capitalisme ni aux cycles économiques, pourtant étudiés dans l'ouvrage (p. 46 pour le premier, p. 85 pour le second).

Annie DUPRAT

Martine Sonnet, Brigitte Keriven, Claude Ghiati, Isabelle Havelange, Bibliographie annuelle de l'histoire de France du ve siècle à 1958. Année 2000, t. 46, CNRS éditions, Paris, 2001, 1054 p., $102 €$.

On ne présente plus cet indispensable outil de travail dont la parution annuelle, depuis près d'un demi-siècle, est régulièrement saluée par les historiens. Les quatre ingénieures du CNRS ("Institut d'histoire moderne et contemporaine ") qui ont réalisé cette quarante-sixième livraison de l'ouvrage à partir " principalement " des collections de la Bibliothèque Nationale de France ont 
recensé 13397 références provenant notamment des dépouillements de 1268 revues françaises et 693 revues étrangères auxquels s'ajoutent 306 volumes collectifs. On sait que le classement thématique des références en neuf grandes rubriques reflète les principales directions de la recherche historique actuelle : "manuels généraux et sciences auxiliaires de l'histoire "; " histoire politique "; " histoire institutionnelle "; " histoire économique "; " histoire sociale "; " histoire religieuse "; " histoire de la France outre-mer "; " histoire de la civilisation "; " histoire locale ".

C'est pourquoi il est toujours instructif de consulter l'" avertissement " liminaire dans lequel les auteurs font part succinctement de quelques commentaires qu'inspirent les statistiques effectuées à partir de ces dépouillements. Pour la première fois, un tableau récapitulant les " formes et origines des travaux " sur les quatre dernières décennies vient pondérer les données reposant sur le seul décompte des notices. On ne sera pas surpris outre mesure de constater que cette prise en compte de la "forme " (ouvrage, articles) fait ressortir l'énorme prépondérance des articles (85\% des entrées) sur les ouvrages : publish or perish, veut le dicton anglais! Par contre, la distinction entre les revues d'" intérêt " général " de niveau universitaire et de large diffusion " et celles d'« intérêt local ou disciplinairement très spécifiques, de diffusion restreinte ", comporte forcément une part de relativité : où situer, par exemple, sur cette grille les Annales de Bretagne et des Pays de l'Ouest? Les auteurs concluent d'ailleurs que cette première esquisse appelle bien sûr " de multiples compléments et nuances comme elle induit une réflexion sur les limites du recensement bibliographique ».

Comme on pouvait s'y attendre, c'est en histoire politique que le pourcentage d'ouvrages (21,3\%) est le plus important par rapport aux articles (78,7 \%), tant en France qu'à l'étranger, alors que l'histoire économique et sociale donne surtout lieu à la production d'articles. Le fait est évident en ce qui concerne l'histoire contemporaine où l'intérêt pour la Troisième République ne se dément pas. Ce sujet qui occupe la première place depuis plusieurs années poursuit son ascension (de $21,7 \%$ en 1996 à $28 \%$ en 2000, pour ce qui concerne uniquement l'histoire politique). Par contre, en histoire moderne, l'intérêt pour les XVII $\mathrm{e}_{-}$ $\mathrm{XVIII}^{\mathrm{e}}$ siècles n'est pas dû à une approche politique, une fois amorti l'effet d'entraînement des anniversaires (bicentenaire de la Révolution; commémoration de l'édit de Nantes...). Durant les quatre dernières décennies, c'est surtout l'histoire sociale qui a bénéficié de cette évolution. Elle s'est développée en renouvelant ses centres d'intérêt : " démographie, vie quotidienne, histoire des femmes et de la vie familiale l'ont successivement innervée ". L'attention du signataire du présent compte rendu a été retenue par le déclin relatif de la période médiévale qui occupait jusqu'alors le troisième rang (tous champs historiographiques confondus) et qui s'efface en l'an 2000 derrière le xvII ${ }^{\mathrm{e}}$ siècle et les années 1815-1851. Toutefois ce recul paraît moins sensible si l'on se reporte aux statistiques relatives à la seule " histoire politique ". S'agit-il enfin là du " retour au politique " annoncé parmi les médiévistes depuis quelques lustres, dans la foulée de l'événementiel, et qui tardait à se manifester?

La croissance remarquable (de $9 \%$ du chapitre " manuels généraux et sciences auxiliaires " en 1960 à près de $26 \%$ en 2000) de la rubrique " historiographie " (plus de 500 notices cette dernière année) atteste de l'approfondissement de la réflexion épistémologique des historiens sur leur discipline, qu'il s'agisse d'expertises répondant aux attentes du corps social ou de retombées de la vague d'" ego-histoire ". Le succès de ce dernier genre historiographique, au même titre que le nombre des Mélanges (34 volumes dépouillés), constitue sans doute une conséquence imparable de l'évolution de la pyramide des âges 
de la corporation. Une étude plus fine des statistiques à moyen terme suggère d'intéressants transferts de sujets entre " périodiques généraux et locaux ". C'est le cas des études de démographie historique qui se cantonnent à présent aux revues des sociétés savantes. D'un autre côté, la stabilité, sur le long terme, de l'histoire religieuse et culturelle ne tient-elle pas, pour une part, à la " relative sur-représentation " des travaux étrangers dans ces domaines (16,5\% de travaux étrangers en histoire religieuse au cours des quatre dernières décennies)?

Comme précédemment, bien entendu, le triple index (index chronologique, index par matières et table des auteurs) qui met près de 300 pages au service de l'utilisateur, orientera celui-ci vers son objet d'étude : thème, personnage, période ou lieu. Un système de renvois permet, en outre, à partir de la référence à un ouvrage collectif, d'en retrouver les diverses contributions, éparpillées par thèmes et par périodes. De même que ce n'est jamais du temps perdu que de flâner au hasard des rayonnages d'une bibliothèque (quand les fichiers électroniques n'y mettent pas le holà!), gageons que le chercheur pourra toujours en parcourant l'ouvrage découvrir, par surcroît, ce qu'il ne s'attendait pas à y trouver. Chacun sait, d'expérience, qu'il n'est pas rare que la consultation d'un titre apparemment sans rapport avec une enquête en cours amène à soulever des questions qui finissent par s'y rapporter.

Bernard MERDRIGNAC

Christian PelRAS, Goulien, commune bretonne du Cap Sizun. Entre XIX siècle et III millénaire, Rennes, PUR, 2001, 486 p.

Le titre surprend : paradoxe d'un regard à la fois restreint géographiquement - Goulien est une petite commune rurale du Finistère sud - et large chronologiquement, qui se rattache au passé et s'évade vers l'avenir. Il suggère pourtant parfaitement l'objectif et la construction de cet ouvrage publié par les Presses Universitaires de Rennes.

La première partie (320 pages) est la reprise - après quelques modifications - de la thèse d'ethnologie soutenue par Christian Pelras en 1965. Le premier objectif de ce travail était d'observer l'adaptation du monde rural à la modernité afin de compléter, en observant un nouveau lieu, les enquêtes menées sur Plozévet. Pour mener cette enquête à bien, Christian Pelras et sa famille ont pris part à la vie de Goulien pendant deux ans. Cette proximité, facilitée par l'apprentissage du breton, a permis à l'auteur des observations fines de la vie rurale. Après une rapide présentation de la commune, il nous les transmet, regroupées en quatre points : les moyens de subsistance, les conditions concrètes de la vie quotidienne, les traditions, les pratiques et les croyances, et enfin la société. Cette monographie est globale puisque le chercheur cerne l'ensemble de la vie quotidienne, cependant les détails fourmillent. La journée de travail d'un paysan, les intérieurs des maisons, l'alimentation, la façon de cuisiner sont décrits avec minutie; ils nous surprennent parfois : pourquoi au Cap Sizun ne conservet-on pas le sang du cochon lors de la tuerie? Ne fait-on pas de boudin? La galette et la crêpe, bien que différentes, sont ici faites l'une et l'autre à base de blé noir, etc. Les pages qui portent sur les réseaux sont riches : sur les liens familiaux et les comportements matrimoniaux bien sûr, mais aussi sur les relations politiques ainsi que sur les rapports d'entraide au travail. Cette analyse, bien 